\title{
Argumente für ein strafrechtliches Stalking-Bekämpfungsgesetz - der Gesetzentwurf des Bundesrates
}

Helmut Fünfsinn

\section{$\S 238$}

Schwere Belästigung (BRatE)

(1) Wer unbefugt und in einer Weise, die geeignet ist, einen Menschen in seiner Lebensgestaltung erheblich $\mathrm{zu}$ beeinträchtigen, diesen nachhaltig belästigt, indem er fortgesetzt

1. ihm körperlich nachstellt oder ihn unter Verwendung von Kommunikationsmitteln verfolgt,

2. ihn, einen seiner Angehörigen oder eine andere ihm nahe stehende Person mit einem empfindlichen Übel bedroht oder

3. andere, ebenso schwerwiegende Handlungen vornimmt,

wird mit Freiheitsstrafe bis zu drei Jahren oder mit Geldstrafe bestraft.

(2) Bringt der Täter das Opfer, einen Angehörigen des Opfers oder einen anderen dem Opfer nahe stehenden Menschen durch die Tat in die Gefahr einer erheblichen Gesundheitsschädigung, so ist die Strafe Freiheitsstrafe von drei Monaten bis zu fünf Jahren.

(3) Auf Freiheitsstrafe von einem Jahr bis zu zehn Jahren ist zu erkennen, wenn der Täter das Opfer, einen Angehörigen des Opfers oder einen anderen dem Opfer nahe stehenden Menschen bei der Tat körperlich schwer misshandelt oder durch die Tat in die Gefahr des Todes oder einer schweren Gesundheitsschädigung bringt.

(4) Verursacht der Täter durch die Tat den Tod des Opfers, eines Angehörigen des Opfers oder eines anderen dem Opfer nahe stehenden Menschen, so ist die Strafe Freiheitsstrafe nicht unter drei Jahren.

(5) In minder schweren Fällen des Abs. 3 ist auf Freiheitsstrafe von sechs Monaten bis zu fünf Jahren, in minder schweren Fällen des Abs. 4 auf Freiheitsstrafe von einem Jahr bis zu zehn Jahren zu erkennen.

(6) In den Fällen des Abs. 1 wird die Tat nur auf Antrag verfolgt, es sei denn, dass die Strafverfolgungsbehörde wegen des besonderen öffentlichen Interesses an der Strafverfolgung ein Einschreiten von Amts wegen für geboten hält.
D ie Frage, ob es eines strafrechtlichen Stalking-Bekämpfungsgesetzes bedarf, kann auf den ersten Blick unschwer mit Nein beantwortet werden. ${ }^{1}$ Die Begründungen sind mit den bestehenden strafgesetzlichen Regelungen, den Normen des Gewaltschutzgesetzes und dem Erfolg der ressortübergreifenden kriminalpräventiven Vorgehensweise $\mathrm{zu}$ leisten. Auf den zweiten Blick lassen diese Begründungen jedoch sehr deutliche Lücken erkennen, die den Bundesrat auf Anregung Hessens veranlasst haben, einen entsprechenden Gesetzentwurf in das Gesetzgebungsverfahren einzubringen. Darüber hinaus hat nunmehr auch die Bundesministerin der Justiz überraschend und entgegen früherer Stellungnahmen einen eigenen strafrechtlichen Gesetzentwurf angekündigt.

1. Überlegungen, die gegen die Implementierung eines strafrechtlichen Stalking-Bekämpfungsgesetzes sprechen könnten

Unbestritten ist, dass Tatbestände des Strafgesetzbuches, die durch ein Stalking-Verhalten verwirklicht werden können, in größerer Anzahl vorhanden sind. Der Tatbestand der Körperverletzung ist dann - aber eben auch nur dann - einschlägig, wenn es zu einer körperlichen Misshandlung oder Gesundheitsbeschädigung kommt.

Da es regelmäßig das Ziel des Täters ist, das Opfer zu einem bestimmten Verhalten - typischerweise zur Aufnahme von Kontakt oder gar einer Beziehung - zu veranlassen, kann auch der Tatbestand der Nötigung (§ 240 StGB) erfüllt sein. Tatsächlich werden allerdings zumeist Schutzvorkehrungen des Opfers hervorgerufen, wie z.B. Sicherheitsmaßnahmen an der Wohnung, die Einrichtung einer neuen Telefonnummer, der Wechsel des Wohnortes oder des Arbeitsplatzes. Auch ein solches Opferverhalten ist zwar Folge des Stalkings, aber gerade nicht intendiert und somit kein Nötigungserfolg im Sinne von § 240 StGB. ${ }^{2}$

Durch Beschimpfungen des Opfers, z.B. wegen einer Untreue oder ablehnenden Haltung, per Brief, Telefon oder EMail, die geeignet sind, eine Person in ihrem sozialen bzw. personalen Geltungsbereich herabzuwürdigen, verwirklicht der Täter den Tatbestand der Beleidigung (§§ 185 ff. StGB). ${ }^{3}$ Adressat einer derartigen Herabwürdigung kann freilich auch der Partner oder ein Beschützer des Opfers sein. Im Nachstellen oder Auflauern allein liegt eine Missachtung des personalen Geltungsanspruchs dagegen selbst dann nicht, wenn der Stalker den ausdrücklichen Wunsch des Opfers, es in Ruhe zu lassen, bewusst ignoriert. 4

Im Übrigen können noch weitere Straftatbestände erfüllt sein, wie etwa die Bedrohung ( $\$ 241$ StGB), die Freiheitsberaubung (§ 239 StGB) und der Hausfriedensbruch (§ 123 StGB).

Darüber hinaus sieht nunmehr auch das seit dem 1. Januar 2002 in Kraft getretene Gewaltschutzgesetz in $\S 1$ nicht 
nur zivilrechtliche Maßnahmen zum Schutz vor Gewalt und Nachstellung vor, sondern erfasst in diesem Paragraphen auch Fälle des Stalkings, also das wiederholte Überwachen oder Auflauern, Verfolgen usw.

Die Folgen der Verstöße gegen diese Vorschrift sind aber nicht die Strafbarkeit, sondern geben allein einem Zivilgericht auf Antrag der verletzten Person, also des Opfers, die Möglichkeit, die zur Abwendung weiterer Verletzungen erforderlichen Maßnahmen zu treffen. ${ }^{5}$ Wenn diese Maßnahmen dann vollstreckbar angeordnet worden sind, kann möglicherweise strafrechtlicher Schutz erlangt werden. Insoweit bestimmt § 4 GewSchG, dass bei Zuwiderhandlungen gegen eine bestimmte vollstreckbare Anordnung - also etwa ein Aufenthalts- oder Näherungsverbot eine Freiheitsstrafe bis $\mathrm{zu}$ einem Jahr oder eine Geldstrafe vom Strafgericht ausgesprochen werden kann. Damit wird allerdings nicht das eigentliche Nachstellen selbst, sondern allein die Unbotmäßigkeit gegenüber der gerichtlichen Anordnung unter Strafe gestellt. ${ }^{6}$

Die bisherige Zusammenarbeit bei der Anwendung des Gewaltschutzgesetzes zeigt, dass konsequente Intervention gegen häusliche Gewalt durch ein koordiniertes Vorgehen der unterschiedlichen Ressorts sowohl auf regionaler Ebene wie auch landesweit der Prävention von häuslicher Gewalt dient. Das Gewaltschutzgesetz wird dabei durch ein polizeiliches Wegweisungsrecht und Betretungsverbot flankiert.

Trotz der aufgezeigten gesetzlichen und tatsächlichen Möglichkeiten des Einschreitens bleibt der Schutz der Opfer aber noch verbesserungswürdig.

\section{Argumente für ein straf- rechtliches Stalking-Bekämp- fungsgesetz}

a) Lücken des Strafgesetzbuchs

Geht man davon aus, dass Stalking letztlich eine Verhaltensweise beschreibt ${ }^{7}$, die dadurch gekennzeichnet ist, dass einer Person fortdauernd nachgestellt, aufgelauert oder auf sonstige Weise Kontakt mit ihr gesucht wird, dann wird deutlich, dass dieses Verhalten so vom Strafgesetzbuch nicht erfasst wird. ${ }^{8}$ Das Verhalten des Täters setzt sich nämlich regelmäßig aus einer Vielzahl zum Teil stark heterogener Einzelhandlungen zusammen, die häufig erst durch ihre Kombination und Wiederholung zu einer unzumutbaren Beeinträchtigung des Opfers werden. Das Spezifikum dieser fortwährenden, sich wiederholenden Belästigung und Nachstellung, also der besondere Eigenwert dieser Verhaltensmuster, wird indessen ebenso wenig wie das zugrundeliegende »Einzelverhalten «, das von der Gesellschaft als sozialadäquat empfunden wird und daher nicht strafbewehrt ist, von einem Straftatbestand umschrieben. ${ }^{9}$ Ist es aber so, dass Stalking gerade dadurch gekennzeichnet ist, dass die Freiheit der Tatopfer und deren Lebensqualität erst durch die Vielzahl von - im Einzelnen oft gar nicht schwerwiegenden Handlungen, primär ihr Andauern oder ihre ständige Wiederholung, nachhaltig beeinträchtigt wird, ohne dass es zwingend zu einer körperlichen Beeinträchtigung kommen muss, dann fehlt hier der strafrechtliche Schutz. ${ }^{10}$ Denn dem besonderen Unwert der genannten Verhaltensweisen kann nur mit einem eigenständigen Tatbestand Rechnung getragen werden, der auch unabhängig davon eingreift, ob zusätzlich mit Gewalt gedroht oder diese sogar angewandt wird. ${ }^{11}$

\section{b) Probleme des Gewaltschutz- gesetzes}

Der strafrechtliche Schutz des Gewaltschutzgesetzes ist lückenhaft. Aus der Sicht des Opferschutzes ist gerade problema- tisch, dass der strafrechtliche Schutz im geltenden Recht unter dem Vorbehalt einer vom Opfer $\mathrm{zu}$ erwirkenden zivilrechtlichen Entscheidung gestellt ist. ${ }^{12}$ Insbesondere in drastischen Fällen kann sich ergeben, dass dem Opfer angesichts der vom Verfolger ausgehenden Bedrohung und der damit verbundenen Ängste der Weg in eine zivilrechtliche Auseinandersetzung versperrt ist. Das Opfer muss also selbst die Voraussetzung schaffen, um später strafrechtlichen Schutz zu genießen. ${ }^{13}$ Auch wenn das Strafrecht dem Opfer verschiedentlich die Initiative zum Anstoß des Strafverfahrens aufgibt, können doch die Opferbelange und die Befangenheit des Opfers in der Beziehung zum Täter viel früher Berücksichtigung finden und insbesondere bei Privatklagedelikten auch sofort eine Strafverfolgung von Amts wegen veranlassen. Es ist damit schwerlich einzusehen, warum der strafrechtliche Schutz gegen Belästigungen erst mit einer zivilrechtlichen Anordnung eintritt. Denn die beeinträchtigende Qualität des Nachstellens als solche ändert sich mit der Entscheidung des Zivilgerichtes nicht. Aus der Sicht des Opfers ist es mithin völlig unerheblich für die Bemessung der Beeinträchtigungen durch den Belästiger, ob zuvor ein Zivilverfahren durchgeführt worden ist oder nicht. Vielmehr kann gerade hier in einer schwelenden Auseinandersetzung viel Zeit verloren gehen, in der das Opfer jedenfalls keinen strafrechtlichen Schutz genießt und hilflos bleibt. ${ }^{14}$

Dies wird besonders augenfällig, wenn der Täter, nachdem gegen ihn eine Schutzanordnung erlassen wurde, sein Verhaltensmuster bewusst dergestalt ändert, dass das Opfer gezwungen ist, jedes Mal erneut eine Schutzanordnung zu erwirken, um strafrechtlichen Schutz zu genießen.

\section{c) Ressortübergreifende kriminal- präventive Zusammenarbeit}

Auch wenn die ressortübergreifende kriminalpräventive $\mathrm{Zu}$ sammenarbeit zur Bekämpfung von häuslicher Gewalt bzw. Gewalt im sozialen Nahbereich und damit auch bei der Bekämpfung von Stalking-Fällen sich ständig verbessert, zeigt diese Zusammenarbeit die Notwendigkeit eines Stalking-Bekämpfungsgesetzes. Gerade der Polizei, die eine große Last bei der Bekämpfung der häuslichen Gewalt zu tragen hat und letztlich die Weichen stellt für eine sinnvolle Anwendung der Gesetze, würde eine große Hilfsmöglichkeit gegeben. Sie könnte nämlich von Anfang an nicht nur als Sofortmaßnahme ein Näherungsverbot oder gar ein Betretensverbot für eine kurze Zeit aussprechen, sondern zugleich die Möglichkeiten eines Strafverfahrens einsetzen, um deutlich zu machen, dass dieses Verhalten nicht nur nicht geduldet wird, sondern dass es auch strafbar ist. ${ }^{15}$ Alle weiteren kriminalpräventiven Akteure in diesem Feld könnten sich ebenfalls auf diese klare Norm berufen und ihre Tätigkeit mühelos legitimieren.

Nach dem Aufzeichnen der Lücken sollte deutlich geworden sein, dass die durchaus positiven Erfahrungen mit dem Gewaltschutzgesetz noch durch die Aufnahme eines StalkingTatbestandes - unzumutbares Belästigen - im Strafgesetzbuch deutlich verstärkt werden könnten. Hier kann die normbildende Kraft des Strafrechts ${ }^{16}$ und die symbolische Ausstrahlung ${ }^{17}$ der Handlungsanleitung des Strafgesetzbuches vor allem auch generalpräventiv genutzt werden.

\section{Entwurf eines Stalking-Be- kämpfungsgesetzes des Bun- desrates}

Der Gesetzentwurf des Bundesrates ${ }^{18}$ möchte einem besseren strafrechtlichen Schutz von »Stalking «-Opfern mit der Einführung spezifischer Straftatbestände gegen die schwere Belästigung (§ 238 StGB-E) Rechnung tragen (vgl. Kasten).

Mit diesem Gesetzentwurf wird nicht nur eine eigenständi- 
ge Strafnorm, die die fortgesetzte Verfolgung, Belästigung und Bedrohung einer anderen Person gegen deren Willen als durchaus schweres, strafwürdiges Unrecht kennzeichnet, geschaffen, sondern zugleich werden Regelungen vorgeschlagen, die eine »Bedrohungsspirale « beenden können. Dies soll neben der Möglichkeit, durch die vorgeschlagene Strafnorm sofort einzugreifen, auch durch die Ergänzung des Haftgrundes der Wiederholungsgefahr in $\S 112$ a StPO erreicht werden, indem eine "Deeskalationshaft « für gefährliche Täter des »Stalking « eingeführt wird.

Abschließend ist darauf hinzuweisen, dass ein eigenständiges »Stalking «-Strafgesetz in einem Spannungsverhältnis steht: Einerseits muss das Gesetz hinreichend konkrete Kriterien für eine Strafbarkeit vorgeben, um dem strafrechtlichen Bestimmtheitsgrundsatz und den praktischen Anforderungen zu genügen. ${ }^{19}$ Andererseits muss der Tatbestand die vielfältigen Erscheinungsformen des "Stalking « möglichst erschöpfend erfassen. ${ }^{20}$ Der Entwurf dürfte diesen beiden Zielvorgaben entsprechen. Er beschreibt die strafbaren Tathandlungen so konkret wie möglich, indem er das Suchen der körperlichen Nähe, die Herstellung des Kontakts mit Kommunikationsmitteln oder die Bedrohung des Opfers oder nahestehender Personen als Tathandlungen aufführt. Soweit darüber hinaus auch andere, ebenso schwerwiegende Handlungen unter Strafe gestellt werden sollen, trägt der Entwurf der Tatsache Rechnung, dass die »Stalking «-Handlungen - wie gesehen - nicht abschließend beschrieben werden können.

\section{Fußnoten:}

1 Die Diskussion um das Phänomen "Stalking « ist bislang eher aus polizeilicher und kriminologischer Sicht geführt worden. Siehe hierzu u.a. Hoffmann, Kriminalistik 2001, 34 ff. und Kriminalistik 2003, 726 ff.; Kube, Kriminalistik 1999, 161 ff.; Füllgrabe, Kriminalistik 2001, 163 ff.; Löbmann, MSchrKrim 85 (2002), 25 ff.; Sieverding, Kriminalistik 2004, 763 ff. Strafrechtlich ist sie von v. Pechstaedt, Stalking-Strafbarkeit nach englischem und deutschem Recht, 1999, angestoßen worden. Während Meyer, ZStW 115 (2003), 249 (259) noch behaupten konnte, dass »Kommentar- und Lehrbuchliteratur zum Thema schweigen«, äußern sich jetzt sowohl Dreher/Fischer, StGB, 52. Auflage (2004), § 223 Rn. 6 als auch Lackner/Kühl, StGB, 25. Auflage (2004), § 223 Rn. 4 knapp zum sog. »stalking «.

2 So zu Recht Meyer, ZStW 115 (2003), 249 (263).

3 Siehe etwa Schönke/Schröder/ Lenckner, StGB, 26. Auflage, § 185 Rn. 2.

4 Meyer, ZStW 115 (2003), 249 (265).

5 Siehe zu den Einzelheiten der Regelung Grziwotz, NJW 2002, $872 \mathrm{ff}$.

6 Vgl. Kerbein/Pröbsting, ZRP 2002, 76 (78).

7 Eine »Legaldefinition « des Begriffes »stalking « existiert noch nicht. Die gegebenen Beschreibungen sind weder eindeutig noch übereinstimmend, vgl. zuletzt Sieverding, Kriminalistik 2004, 763, die zu Recht darauf hinweist, dass dies ein Problem im Umgang mit dem Phänomen darstellt. Siehe auch Rinio, Kriminalistik 2002, S. 531.

8 Löbmann, MSchrKrim 85 (2002), 25 (30); Kerbein/Pröbsting, ZRP 2002, 76 (78); Pollähne, Neue Kriminalpolitik 2002, 5b; Lackner/Kühl, StGB, 25. Auflage, § 223 Rn. 4; Meyer, ZStW 115 (2003), 249 (266).

9 Meyer, ZStW 115 (2003), 249 (267).

10 v. Pechstaedt, a.a.O., S. 132 f.
11 v. Pechstaedt, a.a.O., S. 133.

12 Siehe Kerbein/Pröbsting, ZRP 2002, 76 (78); zu den dogmatischen Ungereimtheiten des $\S 4$ GewSchG vgl. umfassend Meyer, ZStW 115 (2003), 249 (269 ff.).

13 Kerbein/Pröbsting, ebenda.

14 Siehe die in Fußnote 12 Genannten.

15 So deutlich Kerbein/Pröbsting, ZRP 2002, 76 (78).

16 Vgl. zur Aufgabe des Strafrechts LK-Jescheck, StGB, 11. Auflage, Einleitung Rn. 1 ff. und zu seiner Grenze (»ultima ratio«) Jescheck, a.a.O., Rn. 3.

17 Der Begriff "Symbolisches Strafrecht « ist in der Strafrechtswissenschaft eher negativ besetzt, weil damit umschrieben wird, dass dieses Strafrecht weniger auf den Schutz der jeweiligen Rechtsgüter angelegt ist als auf weiterreichende politische Wirkungen wie etwa die prompte Befriedigung eines Handlungsbedarfs; so Hassemer, NStZ 1989, 553 (559). Gleichwohl wird der präventive Gewinn gesehen; etwa Hassemer, a.a.O., 558. Unter Berücksichtigung dieser präventiven Wirkung und des intendierten Rechtsgüterschutzes, der hier konkret den inneren Rechtsfrieden des Opfers betrifft vgl. Meyer, ZStW 115 (2003), 249 (284) - und damit als Freiheitsschutz verstanden werden kann, lässt sich die Sache auch ins Positive wenden. Siehe zum Rechtsgüterschutz allgemein zuletzt Roxin, ZStW 116 (2004), S. 929 ff.

18 BR-Drs. 551/04, BT-Drs. 15/5410.

19 Vgl. zum Bestimmtheitsgebot umfassend LK-Gribbohm, StGB, 11. Auflage, $\S 1$ insbesondere
Rn. 26 ff. m.w.N. sowie Lackner/Kühl, StGB, 25. Auflage, $\S 1$ Rn. 7 zur schwierig zu bestimmenden Grenze zwischen unzulässiger Analogie und zulässiger Auslegung. Zur rechtsstaatlichen und rechtspolitischen Bedenklichkeit unbestimmter Strafbarkeitsvoraussetzungen siehe Naucke, JuS 1989, 862 ff.

20 Einerseits müssen der gesetzliche Tatbestand und damit die Voraussetzungen der Strafbarkeit so konkret umschrieben werden, dass der Einzelne die Möglichkeit hat, sein Verhalten auf die Rechtslage einzurichten und sich Tragweite und Anwendungsbereich des Straftatbestandes erkennen lassen (ständige Rspr. des BVerfG, vgl. etwa BVerfGE 14, 174 und 87, 224). Andererseits dürfen die Anforderungen an die Bestimmtheit nicht übersteigert werden, da ohne allgemeine, normative und wertausfüllungsbedürftige Begriffe »der Gesetzgeber nicht in der Lage wäre, der Vielgestaltigkeit des Lebens Herr zu werden « (BVerfGE 11, 237). Zur Frage der Bestimmtheit einer weit gefassten »Stalking «-Strafvorschrift vgl. Meyer, ZStW 115 (2003), 249 (288), der etwa die Formulierung »ähnliche Eingriffe « für zulässig erachtet.

Dr. Helmut Fünfsinn ist Leiter der Abteilung Strafrecht und Gnadenwesen im Hessischen Ministerium der Justiz.

\section{Vorschläge des Deutschen Juristinnenbundes für ein sinn- volles Vorgehen gegen Stalking}

\section{Dagmar Freudenberg}

$\int 1$ talking ist kein neues Phänomen. Das Verhalten von Stalkern, nämlich das Verfolgen gegen den Willen der verfolgten Person auf welche Weise auch immer, gibt es schon sehr lange. Auch bei der Einführung des Gewaltschutzgesetzes war dieses Phänomen bekannt. Deshalb wurden einige der zu dieser Zeit als Stalking erkannten Handlungen in $\S 1 \mathrm{Ab}$ satz 2 GewSchG erfasst.

In den letzten Jahren ist dieses Phänomen auf Grund einiger Fälle mit prominenten Opfern, aber auch durch die Fokussierung auf die häusliche Gewalt in den Blickpunkt geraten. Ein großer Teil der Fälle von Stalking spielt sich im
Bereich der privaten oder der privat gewünschten Beziehungsebene ab.

Einigkeit besteht dabei darin, dass die Opfer von Stalking unter den Schutz der Gesellschaft gestellt werden müssen. Ein Dissens besteht jedoch über den richtigen Weg zu diesem Schutz. 